\section{Park Systems}

With offices and distribution partners on every continent and a history of unrivaled AFM product innovation, Park Systems is a premier global AFM technology solution provider.

Park Systems offers the broadest line of AFM from research grade to fully automated for researchers and industry engineers in the chemistry, materials, physics, life sciences, and semiconductor and data storage industries. Park's wide range of AFM products are used for research at virtually all major universities and national labs and semiconductor companies world-wide have partnered with Park Systems to fulfill their nanoscale microscopy needs, including the top five manufacturers.

Park Systems has remained the leading innovator in nanoscale microscopy and metrology throughout its long history and continues to invest in the development of new emerging technologies. Products from Park Systems are used by some of the most notable researchers and corporations across the globe and are valued for their many unique and patented AFM designs.

\section{Atomic Force Microscope Products}

Park NX10-The easiest to use and most accurate AFM with Accurate XY Scan by Crosstalk Elimination, Accurate AFM Topography with Low Noise Z Detector, Best Tip Life, Resolution and Sample Preservation by True Non-Contact ${ }^{\text {mix }}$ Mode and Park SmartScan ${ }^{\text {mw }}$ - AFM operating software versatile enough to empower both novices and power users alike toward great nanoscale research. Park NX10 provides accurate measurement at highest nanoscale resolution than any other products in its class. It allows you to obtain sample images and its characteristic measurements true to its nano structure thanks to its flat, orthogonal, and linear scan measurements by its unique AFM architecture: independent XY and Z, flexure based scans. Park's unique True Non-Contact ${ }^{\mathrm{mix}}$ mode provides you with the sharpest images, scan after scan without declining resolution.

Park NX12-The SICM of Park NX12 is the next generation nanoscale microscope for life science. Similar to Scanning Tunneling Microscopy (STM) operating in ambient air, the Park SICM operates in liquid without making physical contact with the sample. Electrodes on either side of the sample and pipette produce ionic current that flows through the surrounding solution. A sensor measures the current flow, which decreases as the distance between the pipette and sample becomes smaller, and monitors the distance between the pipette and the sample to obtain the topology.

Park NX-Hivac- Park NX-Hivac allows failure analysis engineers to improve the sensitivity and resolution of their measurements through high vacuum SSRM. Because high vacuum scanning offers greater accuracy, better repeatability, and less tip and sample damage than ambient or dry $\mathrm{N} 2$ conditions, users can measure a wide range of dope concentration and signal response in failure analysis applications. The NX-Hivac lets you scan a sample in just five steps: Scan, lift cantilever, move motorized stage to user defined coordinate, approach, and repeat. This boosts productivity enormously and reduces required user input to the absolute minimum.

Park XE7-Park XE7 is the most affordable AFM used in research world-wide at leading institutions. Built with the proved Park AFM technology, with reliable and accurate results, this versatile AFM has been used to publish thousands of research papers in a wide variety of industries and applications.

Park NX12 Bio-Reliable and repeatable Nano Bio-imaging for better experimental verification featuring exclusive non-invasive SICM to preserve naïve morphological information of soft bio-materials; excellent imaging repeatability in automatically programmed and running software; accurate height/depth analysis from 3D structure measurements

Park Systems, Inc. is a global manufacturer of Atomic Force Microscopes and a pioneer in AFM Nanometrology. Park's expansive global distribution network includes U.S. headquarters in Santa Clara, CA and a new office at SUNY Polytech in Albany NY, research and manufacturing in Seoul, South Korea, and regional offices in Japan, Singapore, Germany, India, China and Mexico, as well as a vast network of distribution partners throughout Europe, Asia, and the Americas.

Park AFM metrology solutions are designed with a focused effort on customer needs and performance requirements with a steadfast commitment to superior customer service. Park's NanoScience Labs are available to conduct training, prepare samples and assist with customer needs and are used for AFM demos and workshops world-wide.
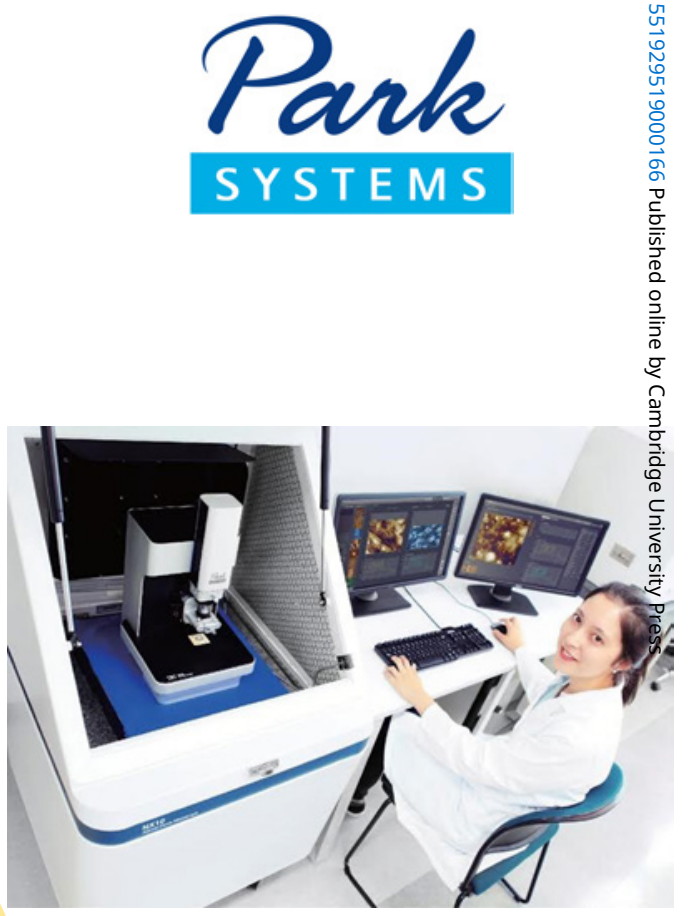

\section{How to find us}

\author{
Park Systems \\ 3040 Olcott St. \\ Santa Clara, CA \\ Tel: 408-986-1110 \\ Email: inquiry@parksystems.com \\ www.parksystems.com
}

